\section{Self-Assembly of DNA into 3D Nanostructures Facilitated with caDNAno Tool}

DNA has proven to be a versatile building block for the fabrication of nanostructures using bottom-up strategies. The design and self-assembly of DNA into twodimensional, megadalton structures have been demonstrated with a strategy that uses multiple-kilobase single strands of DNA that act as scaffolds, which fold into a flat array of antiparallel helices after interacting with hundreds of oligonucleotide strands conceptualized as staples. Recently, W.M. Shih and co-researchers from the Dana-Farber Cancer Institute, Harvard Medical School, and Harvard University have extended this method to the design and fabrication of three-dimensional (3D) nanostructures that are formed as pleated layers of helices constrained to a honeycomb lattice. Shih and co-researchers designed and assembled DNA nanostructures approximating a variety of shapes with precisely controlled dimensions ranging from $10 \mathrm{~nm}$ to $100 \mathrm{~nm}$.

Shih and co-researchers described their honeycomb pleat-based assembly strategy in a letter published in the May 21 issue of Nature (DOI: 10.1038/nature08016; p. 414). As shown in Figure 1, double helices consisting of scaffold strands and staple strands create an unfolded, two-dimensional form of the target shape. Helices that are depicted as adjacent in the conceptual folding intermediate presented in Figure 1 are connected by a mixture of staple and scaffold crossovers, while the helices that are adjacent in the final structure but not in the conceptual folding intermediate are connected only by staple crossovers (both staple and scaffold crossovers are phosphate linkages). The researchers likened the first step in the design process to sculpting a shape from a crystalline block; unwanted DNA helices are carved away from the honeycomb lattice of antiparallel helices. Next, scaffold crossovers at a subset of allowed positions are introduced to create a singular scaffold path that visits all remaining duplex segments. Staple crossovers are then added at posi-

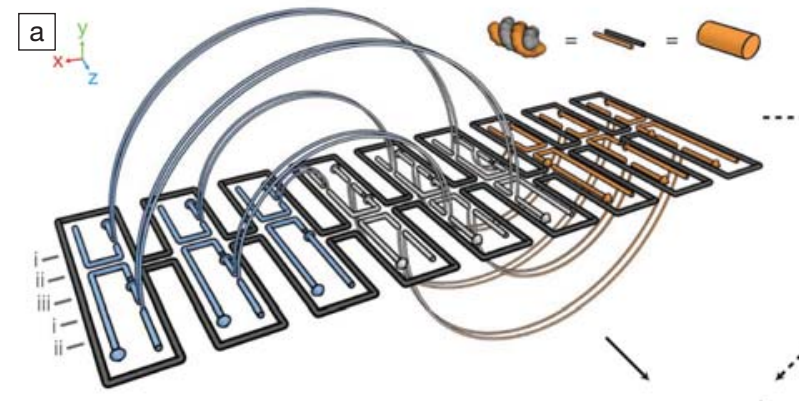

C
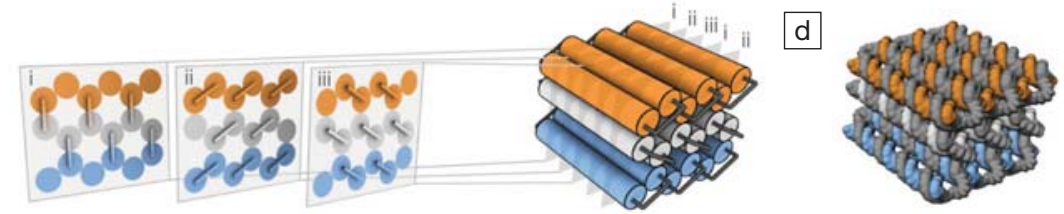

Figure 1. (a) An unrolled two-dimensional schematic of the target shape. Grey scaffold strands and orange, white, and blue staple strands form double helices that run parallel to the $z$-axis. Crossovers between adjacent helices are made with phosphate linkages, while stable crossovers (shown as semicircular arcs) bridge different layers. (b) Partial folding is illustrated with a conceptual intermediate model composed of cylinders. (c) A fully folded target shape, which also shows cross-sectional slices (i-iii) of parallel helices arranged in a honeycomb pattern in the $x-y$ plane. (d) An atomistic model of the target shape. Reproduced by permission from Macmillan Publishers Ltd: Nature 459 (7245) (2009) p. 414, @2009.

tions consistent with the target shape and with other structural considerations. Unpaired scaffold bases are introduced at the ends of helices to control multimerization. The researchers developed caDNAnoa design program with a graphical-user interface- to assist in honeycomb-pleatedorigami design. The researchers said that, after a one-day caDNAno tutorial, inexperienced researchers will be able to generate base sequences for DNA nanostructures.

The researchers used a one-pot reaction to assemble the DNA nanostructures. A mixture of $10 \mathrm{nM}$ scaffold strands (derived from the single-stranded genome of the M13 bacteriophage) and $50 \mathrm{nM}$ of each oligonucleotide staple strand, together with salts and buffer, were subject to a thermal-annealing ramp followed by cooling for $80 \mathrm{~min}$. from $80^{\circ} \mathrm{C}$ to $60^{\circ} \mathrm{C}$, followed by a 173-h cooling cycle, from $60^{\circ} \mathrm{C}$ to $24^{\circ} \mathrm{C}$. The DNA nanostructures were purified with electrophoresis and imaged using transmission electron microscopy.
The researchers fabricated nanostructures approximating various 3D shapes: a monolith, a square nut, a railed bridge, a slotted cross, and a stacked cross. Programming staple strands to bridge separate scaffold strands will yield hierarchical DNA nanostructures.

The researchers said, "Three-dimensional DNA nanostructures should expand the range of possible applications with an increased range of spatial positioning that is not accessible by flat structures, including those requiring encapsulation or space-filling functionalities, as in many natural biosynthetic machines that use three-dimensional scaffolding to control assembly of complex products. Similar capabilities for synthetic machines are thus more accessible with this convenient, generalizable facility to fabricate customshaped three-dimensional structures from DNA."

STEVEN TROHALAKI

\section{Gold Nanorods Enable Five- Dimensional Optical Recording}

Five-dimensional optical recording has the capability to drastically increase optical storage capacity. Current available storage techniques, such as those involving DVDs and blu-ray, only make use of two dimensions. Researchers P. Zijlstra, J.W.M. Chon, and M. Gu, at the Centre for Micro-Photonics at Australia's Swinburne
University of Technology, have recently developed a single method utilizing gold nanorods that combines multiple recording domains to achieve optical recording in five dimensions. The technique combines the three spatial domains with those of polarization and wavelength to potentially increase storage capacity by orders of magnitude. The technology also has applications in encryption.
As reported in the May 21 issue of Nature (DOI: 10.1038/nature08053; p. 410), the new recording media based on gold nanorods allows for five-dimensional optical recording by using longitudinal surface plasmon resonance. Within a single recording layer, the gold nanoparticles were shown to have the ability to store nine information states. These nine states come from using three different 